\title{
Oral Dysesthesia, CTCAE
}

National Cancer Institute

\section{Source}

National Cancer Institute. Oral Dysesthesia, CT CAE. NCI Thesaurus. Code C143716.

A disorder characterized by a burning or ting ling sensation on the lips, tongue or entire mouth. 